is of alternating intensity and the disappearance of one or more stationary strips will occur when the sensation produced, at a spot on the retina where the stimulus was at maximum intensity, reaches one of its minima. The sensation produced in adjacent parts of the retina, where the stimulus is at its minimum intensity, will then be approximately at maximum intensity; there will thus be very little difference of intensity over an area corresponding to the distance between two alternate bright strips. In general only one bright strip will disappear in this way at any given instant.

All the phenomena observed receive a satisfactory explanation on the basis of the general form of the hypothesis usually adopted to account for the phenomena observed when a moving stimulus of constant intensity travels across the retina. They thus support the hypothesis that these phenomena are largely due to the temporal course of the sensation produced by a stimulus of short duration, although spatial induction may inhibit some part of the course of sensation. This latter factor would appear to act with alternating intensity, and so to be due to the whole of the bright part of sensation, and not merely to that in parts of the retina immediately adjacent to that where inhibition took place.

The unpleasant flickering is not to be confused with that observed when a narrow object (such as a billiard cue), illuminated by an A. C. lamp, is rapidly moved to and fro laterally. This is due entirely to light and dark strips seen in the primary band. It may, however, contribute to the nervous irritation sometimes produced under these conditions, or which some people find (probably due to eye movements) with A. C. lighting under ordinary conditions.

\title{
REFERENCE
}

1. MacDougall, Brit. Jl. of Pysch., Vol. I, 1904; NAgel, Handbuch der Physiol. des Menschen; PARsons, Colour Vision, London, 1924.

\section{ROYAL LONDON OPHTHALMIC HOSPITAL (Moorfields)}

IN connection with the opening of the new Lecture Hall on October 1 , the following has been contributed by a correspondent.

The Out-Patient Department, Library, Lecture Room, Museum, and Pathological Laboratory were erected in 1898. The Bacteriological Laboratory was added in 1907. In 1925 Sir William Lister built and equipped a smaller lecture room for clinical demonstrations. 
The Out-Patient Department had for many years been found inconvenient in use and inadequate for the increasing number of patients attending each morning. Moreover, since the original department had been built there had been elaboration in the services rendered to patients, and it had been found increasingly difficult to accommodate them all. Old students will remember the confusion in the consulting room, and refraction room; they will also call to mind the number of patients that collected around the room for minor surgery and the crowding of the third HouseSurgeon's department. More recently a large L.C.C. clinic had been set up, and an Almoner's department established. There was inadequate accommodation for perimetry, and the introduction of the Bjerrum Screen had called for further accommodation.

Two years ago, in 1933, all these buildings were demolished and further ground space was obtained.

It had been felt that when the new buildings were raised to take the place of the old some attempt should be made to improve on the previous plan that had become stereotyped in all ophthalmic hospitals; several years of thought had been given to the matter and many sets of plans had been made, to be rejected or modified in the attempt to found not only a more useful building but one of artistic merit.

The first point that was decided was that each surgeon should have a separate consulting room with suitable dark room accommodation, for the investigation of his cases, immediately at hand. The second was that there should be proper facilities for the refraction of the eyes of patients, with the patient and surgeon at ease, in quiet surroundings, and the refraction rooms separate from one another. . It will be seen that by an ingenious arrangement seventeen refraction units have been set up.

Another point decided upon was that new patients coming to the hospital should be 'sifted' and thus, by a judicious assembling of patients, both the patients' and surgeons' time would be economized. All this has been arranged for in the new department.

All departments had increased in size, and among others, accommodation was needed for the medical, dental, and venereal clinics, as well as those for X-ray examination, physio-therapy and orthoptic training, many of which had not been in existence when the old buildings were erected. Further, an elaborate L.C.C. department with its officers and secretarial staff was housed, and more recently a large Almoner's department established. Arrangements have been made for all their requirenents in the
new buildings.

So far as the Out-Patients' department and Medical School are concerned, the building is arranged in three floors. 
Taking the ground floor first: Patients are admitted from Peerless Street at the back of the hospital, and enter a crush room, from which they pass into the general waiting room to be registered in the case of new patients, and receive their letters if old patients from the letter store. They are then arranged in forms allotted to the various surgeons and passed on to the desks where the sifters are at work. Here all new patients are divided into categories : refraction cases passing to a waiting room by the side of the refraction hall; minor surgical cases to a waiting room, and so to the rooms where the third House-Surgeon and OutPatient Sister are at work. Other cases are sent to a waiting room outside the surgeon's consulting room to be interviewed by him in turn.

The refraction department is a new venture : it consists of a large hall, along each side of which are grouped seventeen cubicles, the outer doors of which communicate with the waiting hall, and the inner doors enable the patient to look across the hall at a test-type on the opposite side which is controlled from the cubicles. No one enters the hall itself. In these cubicles, the patients comfortably seated, are tested for spectacles, and when the examination is completed, are returned to the waiting room outside, and so on to the spectacle room.

Of the minor surgical cases (cysts of the eyelids, foreign body cases, etc.) a proportion are sent to the surgeon that they may be of value as teaching material.

The surgeons' consulting rooms show these general arrangements : they are about $25 \mathrm{ft}$. long and $14 \mathrm{ft}$. wide, and behind the surgeons' desks there is a common dark room for a group of three consulting rooms, whereas the two end rooms have their dark rooms separate, but immediately available. Owing to the general use of the electrically lit ophthalmoscope there is no need for the numerous divisions which were in use in the past. With the new arrangements there are suitable ophthalmic brackets conveniently placed, as well as accommodation for slit-lamps and perimeters.

The result of this is a quiet and wholesome atmosphere which makes for better and more rapid work, as well as very much less fatigue among those working in the departments.

All new cases pass to the Lady Almoner on the way to the dispensary, and patients leave the hospital by separate exits into Peerless Street.

The X-ray department has been improved and enlarged.

There a separate refreshment rooms for patients and students on the ground floor.

On the first floor which is reached by stairway and lift, there 
is an extensive physio-therapeutic department and orthoptic clinic. These complete the arrangements for clinical work. Here too are the new Museum and Library, where there is enough shelf room to allow for very considerable expansion. In the Library there are a series of alcoves for readers.

On the second floor is a new Bacteriological laboratory and the Treacher Collins Pathological laboratory. Here also is the main Lecture Hall which will hold sixty students comfortably seated, and will permit the accommodation on occasions, such as clinical meetings of the Ophthalmological Society, of any number up to one hundred. Here is the epidiascope. There is also a small Lecture Room for classes up to twenty in number.

The above description applies only to the new Out-Patients department and Medical School. There are many other improvements in the hospital itself.

On the fourth floor there is a suite of thirty-one private wards of one bed each. This unit is complete with theatre and sterilizing plant. To each cataract ward in the general building there is attached a contributary ward, containing five beds, each surrounded by curtains.

The old building is undergoing re-organization. There are now two operating theatres as well as extra examination rooms and a re-arrangement of space so that now the hospital accommodates two hundred in-patients.

\section{NORTH OF ENGLAND OPHTHALMOLOGICAL SOCIETY Twenty-first Anniversary Meeting}

THE North of England Ophthalmological Society held its 21st anniversary meeting at Manchester on Friday, October 18. The morning session was occupied by two papers and the showing of Mr. Percival Hay's film of the ophthalmological tour. Unfortunately there was not time to show the whole film, but the part exhibited gave an excellent impression. of what must have been a very interesting experience for those who took part in it.

Mr. Jefferson took as the title of his paper, "The Radiology of the Optic Foramina" and explained the usefulness of such radiograms in the diagnosis of certain tumours. He then gave a fascinating demonstration of the uses of thorotrast in obtaining $\mathrm{X}$-ray pictures of the intra-cranial vessels and of the ventricles of the brain. The clinical application of these methods was also 